\title{
El negocio del cuero en el Río de la Plata a fines del siglo XVIII
}

\author{
The cowhide business in the Rio de la Plata at the end of the 18th \\ century
}

\author{
Nicolás Alberto Biangardi \\ Maximiliano Camarda
}

RESUMO

Este trabajo pretende efectuar una síntesis que muestre un panorama completo de todo el circuito de la producción y comercialización de los cueros vacunos en el Río de la Plata a finales del siglo XVIII. Es decir, un sumario de todas las actividades económicas y los agentes que intervenían desde la matanza del animal hasta la estiba del cuero dentro de la embarcación que lo llevará a través del océano Atlántico, al mercado consumidor. Al mismo tiempo, se incluirán los resultados de recientes investigaciones sobre la circulación de los cueros dentro de la región y su posterior exportación y sobre los agentes involucrados en esas actividades. De esta manera, se busca mostrar la complejidad de la producción y comercialización de los cueros a fines del siglo XVIII, que los agentes identificados se pueden incluir en distintas categorías ocupacionales y, además, destacar que la mayor parte de la población estaba vinculada de alguna manera al negocio de los cueros vacunos.

Palavras chave: Cueros- Rio De La Plata. Siglo XVIII.

ABSTRACT

This work aims to make a synthesis starting to show a complete picture of the entire circuit of production and marketing of cow hides. That is, of all economic activities and agents that were involved from the leather extract of the animal until stowage leather inside the boat carrying him through the Atlantic Ocean, the consumer market. Also, the results of recent research on the circulation of hides within the region and subsequent export and agents involved in such activities will be included. Thus, it seeks to highlight the complexity of the production and marketing of hides in the late eighteenth century, that the identified agents, included in various occupational categories and, finally, show that most of the population was linked in some so the business of cowhides.

Keyword: Leather- Rio De La Plata. Century XVIII.

"Instituto de Investigaciones en Humanidades y Ciencias Sociales (IdIHCS) - Universidad Nacional de La Plata (UNLP) 


\section{Introducción}

La segunda mitad del siglo XVIII fue un momento de profundos cambios en la región Río de la Plata(JUMAR, 2012; JUMAR; BIANGARDI, 2014). En ese momento se produjo un aceleramiento del crecimiento secular de la región que se verifica en un aumento demográfico exponencial y en una constante expansión territorial. Estudios recientes han destacado el lugar de la producción de cueros vacunos y su posterior exportación en el nivel de actividad económica y el crecimiento de la región Río de la Plata.

Por un lado, María Inés Moraes destacó el lugar de la producción de cueros para la exportación dentro del producto agrario de la región. La autora muestra que el aporte de esa actividad llegó a superar el 50\% del total de lo producido, en valores, por la economía agraria regional en las décadas de 1770 y 1780 (MORAES, 2014). Por otro lado, Fernando Jumar sostiene que la importancia de los cueros vacunos dentro de la exportaciones del Río de la Plata radicaba en el hecho de que éstos se producían en su mayor parte en la región, en cambio los metales preciosos que se enviaban a Europa eran el resultado del total de las transacciones comerciales que se realizaban en todo el virreinato (JUMAR, 2012).

En este sentido surge la necesidad de estudiar con detenimiento toda la cadena de actividades que intervenían en la producción y comercialización de esos cueros. A primera vista, la operación de extraer el cuero de un animal y trasladarlo parece relativamente sencilla. Sin embargo, las diferentes actividades involucradas en la producción de cueros vacunos y su comercialización en las campañas rioplatenses del siglo XVIII dibujan una imagen bastante más compleja que la que surgía de las suposiciones iniciales. Esto se debe a que no existía una sola manera de producir esos cueros, ni de trasportarlos, a que se requería de cierto conocimiento para hacerlo, a que su comercialización diferenciaba claramente entre el comercio interno y el exportador y a que los cueros tenían distintos destinos. En su conjunto, estas actividades económicas ocupaban un porcentaje significativo de la población formando parte de la vida cotidiana de los habitantes de la región.

Muchos trabajos han estudiado algunas de estas actividades desde distintas perspectivas ${ }^{1}$. La mayor parte de las investigaciones llevadas a cabo se han enfocado solamente en una parte del problema. En general, en el comercio ultramarino o en la producción agropecuaria. En este trabajo partiendo de los datos disponibles en esa bibliografía, se pretende efectuar una síntesis que muestre un panorama completo de todo el circuito de la producción y comercialización de los cueros vacunos. Es decir, de todas las actividades económicas y los agentes que intervenían desde la matanza del animal hasta la estiba del cuero dentro de la embarcación que lo llevará,

${ }^{1}$ Por razones de espacio solamente se mencionaran estos trabajos ya que un balance de la contribución de cada texto y una revisión de las críticas y debates requeriría de un artículo dedicado exclusivamente a ese propósito. Las vaquerías corambreras fueron estudiadas por: Saguier (1991), Pérez (1996) y Moraes (2011). La producción de las estancias de la campaña de Buenos Aires y de otras partes de la región fueron tratadas por varios trabajos entre los que se destacan: Mayo (1995), Amaral (1998) y Djenderedjian (2003). La circulación interna de los cueros aparece en: Garavaglia (1999), Gelman (1998), Sovarzo y Errecart (2013), Biangardi (2013) y Biangardi (2015a). Por último, la exportación de los cueros en Jumar (2000, 2008), Silva (1993), Moutoukias (1995), Rosal y Schmit (2004) y Camarda (2015). 
a través del océano Atlántico, al mercado consumidor. Al mismo tiempo, se incluirán los resultados de recientes investigaciones sobre la circulación de los cueros dentro de la región y su posterior exportación y sobre los agentes involucrados en esas actividades. De esta manera, se busca destacar la complejidad de la producción y comercialización de los cueros a fines del siglo XVIII, que los agentes identificados se pueden incluir en distintas categorías ocupacionales y, además, mostrar que la mayor parte de la población estaba vinculada de alguna manera al negocio de los cueros vacunos.

\section{Producción y circulación de cueros dentro de la región}

Producción de los cueros: coexistencia de las vaquerías corambreras y la ganadería de rodeo

Durante todo el siglo XVIII coexistieron dos formas de producir cueros en las campañas rioplatenses. Éstas se correspondían a dos actividades económicas dedicadas a la explotación de ganado vacuno (las vaquerías corambreras y la ganadería de rodeo) que diferían en muchos aspectos: su localización geográfica, sus objetivos, el tipo de mano de obra que utilizaban y la organización de las tareas.

Las vaquerías corambreras eran expediciones de caza de ganado vacuno salvaje, que en esa época se denominaba como cimarrón, con el objetivo de extraer la mayor cantidad posible de cueros. Su localización, que dependía de la ubicación geográfica de las reservas de ganado cimarrón, fue cambiando a lo largo del tiempo a medida que la población crecía, se expandía la ocupación de tierras y la ganadería de rodeo. De esta manera, las vaquerías se iban alejando cada vez más de las ciudades portuarias de la región. Durante las décadas finales del siglo, los focos corambreros se hallaban en las tierras situadas al norte del río Negro en la Banda Oriental.

Existieron distintos mecanismos institucionales que buscaron regular esta actividad para, de esa manera, intentar restringir el acceso a los circuitos legales de comercialización. Así durante el primer boom exportador del siglo XVIII el acceso legal al ganado cimarrón era otorgado mediante acciones de vaqueo por el cabildo de Buenos Aires (JUMAR, 2012). En la segunda mitad del siglo las condiciones cambiaron ya que se consideró que todo el ganado existente era alzado y no cimarrón. Esto dio lugar a una disputa por la propiedad del ganado entre distintos agentes económico. Por ende, los mecanismos institucionales para su apropiación legal dependieron de quién reclamaba ese bien. Esencialmente en ese momento existieron dos maneras. En primer lugar, entre 1767 y 1784 se faenaron cueros en las tierras en torno al río Yi y Negro mediante contratos con las administraciones de las misiones de Yapeyú y San Miguel (SARREAL, 2009). En segundo lugar, los agentes con residencia en las ciudades portuarias de la región hicieron denuncias de grandes extensiones de tierras realengas. Esto les permitió ingresar los cueros producidos en las vaquerías como propios argumentando que 
se trataba de ganado alzado de sus estancias donde, habitualmente, mantenían un pequeño rodeo. Paralelamente también había faenas clandestinas que no respetaban los mecanismos institucionales creados para la apropiación legal del ganado y que lograban colocar su producción en los circuitos de comercialización ${ }^{2}$.

Las vaquerías eran empresas que requerían una organización previa y un cierto grado de especialización de sus trabajadores. Por lo general, el corambrero proporcionaba las herramientas de trabajo y raciones de yerba y tabaco. Los vaqueros y peones, por su parte, trabajaban a destajo y utilizaban sus propios caballos. Eduardo Saguier afirma que la paga por cuero incluía el costo del desgaste de la tropilla de caballos (SAGUIER, 1991). Las tareas se dividían en: vaqueros desjarretadores, peones desolladores, barraquero, sebero y peón caballerizo (PEREZ, 1996). En cada vaquería había un promedio de dos o tres vaqueros y de tres a diez desolladores. Los vaqueros se encargaban de desjarretar y acodillar al animal. Los peones desolladores cuereaban el vacuno y procedían a estaquear y marcar el cuero. Luego el barraquero recogía, desgarraba, clasificaba, apilaba y prensaba cada cuero (SAGUIER, 1991).

La ganadería de rodeo, en cambio, aprovechaba el hecho de que el vacuno es un animal que se desplaza poco y se acostumbra a permanecer en un mismo sitio, es decir que se aquerencia. Una vez recogido, el ganado era trasladado hasta una aguada de la estancia, cuando se distanciaba un poco era rodeado y vuelto a llevar al lugar elegido y en unos tres o cuatro meses ya tendía a volver a su querencia cada vez que se alejaba. Por esta razón eran apreciados los campos que tenían acceso al agua y, especialmente, los que contaban con "rincones" (por ejemplo, donde se unen dos arroyos o un arroyo y una laguna) (GIBERTI, 1986). Además de la vigilancia y el rodeo del ganado, en las estancias se realizaban una importante cantidad de actividades, algunas permanentes y otras temporales. La parición tenía lugar de marzo a septiembre, la yerra se realizaba a mediados de invierno y la doma de potros de febrero a abril. También se procedía a la castración de los machos jóvenes, la esquila de ovejas, la cría de mulas y el exterminio de perros cimarrones (GARAVAGLIA, 1999).

Si bien los dos sistemas de explotación del ganado vacuno coexistieron, el agotamiento de las reservas de ganado cimarrón alejaba cada vez más de las ciudades portuarias el espacio donde se realizaban las vaquerías, modificando así sus costos de transporte y los circuitos por donde tenían salida los bienes producidos. Ese espacio liberado, por decirlo de alguna manera, era ocupado por nuevas estancias en un paulatino proceso de expansión territorial que la región vivió de manera ininterrumpida a lo largo de todo el siglo XVIII.

Además de las estancias, en las zonas cercanas a los puertos o embarcaderos del río existieron otros dos tipos de establecimientos que producían cueros y otros derivados del ganado vacuno: los mataderos y los saladeros. Ambos dependían de la provisión de animales por parte de las estancias y su principal objetivo era la producción de carne, ya sea fresca o salada. Aunque estos establecimientos fueron importantes porque propiciaron un aprovechamiento más

${ }^{2}$ Inevitablemente esta situación generaba un conflicto de intereses entre los productores y los comerciantes a los que las faenas clandestinas de cueros les convenían, ya que abarataban el precio de ese bien que buscaban adquirir. 
integral de cada cabeza de ganado sacrificado, el volumen de su producción de cueros no tuvo un peso relevante ${ }^{3}$. La mayor parte de los animales continuaron siendo carneados durante las vaquerías y en las estancias.

Al tratarse de una época pre estadística no se cuenta con fuentes directas para evaluar el nivel de producción de este sector de la economía, más allá de la contabilidad de algunas estancias aisladas. Los datos de la recaudación del diezmo, por si mismos y sin complementar con información proveniente de otras fuentes, tampoco permiten acercarse a una estimación probable 4 . Por ende, la mejor opción para esto es recurrir al estudio de la circulación de esos bienes para tener un indicador indirecto sobre esas actividades económicas.

Por ejemplo, el estudio de la circulación de cueros, sebo y grasa que confluían en la ciudad de Montevideo entre 1784 y 1797 reveló indicios sobre los niveles de producción, su estacionalidad y sobre la incidencia de cada una de las dos actividades dedicadas a la explotación del ganado vacuno (BIANGARDI, 2015b). En primer lugar, se pudo constatar que desde 1785 hasta mediados del 1788 las introducciones de cueros se mantuvieron en niveles bajos (menos de diez mil cueros mensuales), comenzando un crecimiento en los meses finales de 1788 hasta situarse por llegar a superar de los 35-40 mil cueros mensuales en los meses de mayor circulación de los años 1794-1796. En segundo lugar, si bien los cueros llegaban a la ciudad durante todo el año, su arribo se intensificaba considerablemente en los meses primaverales y disminuía de manera significativa durante el invierno. Además, la importancia de los cueros orejanos (es decir sin marca) en las partidas que llegaban en los meses de mayor tráfico permite entrever la influencia de las vaquerías corambreras en esa estacionalidad tan marcada ${ }^{5}$.

\section{Circulación de los cueros: transporte y comercialización en la campaña}

Una vez que los cueros estaban en condiciones eran trasladados hasta las ciudades portuarias. Los cueros vacunos tenían un volumen considerable y, en general, debían ser transportados varios kilómetros hasta llegar a su lugar de almacenamiento ${ }^{6}$. Sin embargo, su precio por peso era relativamente bajo si se lo compara con el de otras mercancías. En consecuencia, su valor era escaso con respecto al volumen que ocupaban en el medio de transporte utilizado y esta desfavorable relación valor/volumen determinaba el lugar central del costo de transporte en el precio final y en las condiciones de comercialización. Esto influía tanto en la circulación de este bien dentro de la región como en su exportación.

\footnotetext{
${ }^{3}$ Apenas el 2,6\% de los cueros que llegaron a Montevideo entre septiembre de 1784 y marzo de 1790 fueron producidos en mataderos. Biangardi (2015a, p. 69).

${ }^{4}$ Para un balance del uso de los diezmos en las investigaciones sobre la producción agropecuaria rioplatense véase: Biangardi (2015b).

${ }^{5}$ Los cueros orejanos eran las pieles que carecían de marca. En algunos años estos cueros tuvieron una participación importante dentro los cueros que llegaban anualmente, llegando al extremo de que en 1793 constituyeron el $63 \%$ del total. (BIANGARDI, 2015a, p. 75).

${ }^{6}$ El tamaño de los cueros variaba según el animal, especialmente había diferencias entre si era de vaca o de toro. Pero para tener una idea aproximada un cuero de vaca mide más de 32 pies cuadrados, es decir 3 metros cuadrados.
} 
El transporte de los cueros desde el lugar de producción hacia las ciudades portuarias podía hacerse de dos formas: de manera terrestre en carros o carretas tirados por bueyes o vía fluvial en diferentes tipos de embarcaciones ${ }^{7}$. Aunque en teoría era más barato y, por ende, preferible, el transporte fluvial no siempre era posible. Lo más común en cambio era el transporte terrestre que se efectuaba en carros tirados por bueyes. Un carro colmado de cueros podía transportar entre 80 y 100, y en algunos casos 110, cueros dependiendo del tamaño de los mismos (BIANGARDI, 2015a). Teniendo en cuenta los miles de cueros que circulaban y que no todos los carros y carretas transportaban solamente cueros ni se encontraban siempre llenos, es sencillo imaginar la importancia de ese tráfico terrestre.

Ahora bien, no todos los productores podían transportar por sus propios medios los cueros hasta el mercado urbano. Los pequeños productores que no disponían de carros y bueyes debían vender sus excedentes a los pulperos asentados en el pago o a los mercachifles que recorrían la campaña vendiendo mercancías. Esa situación determinaba la comercialización de estos bienes en la campaña, mientras que los grandes y medianos productores llevaban los cueros directamente al mercado urbano.

En consecuencia, los pulperos cumplían una función de intermediarios entre los pequeños productores y los mercados de las ciudades portuarias. Jorge Gelman afirma que la presencia de varios de estos establecimientos en un lugar constituía un freno a la explotación mercantil de campesinos y peones ya que la competencia imposibilitaba que un pulpero individual impusiera condiciones a sus clientes (GELMAN, 1998). En este sentido, se puede observar que existía una mayor cantidad de pulperías en las cercanías de los poblados. En cambio, en los parajes más alejados las posibilidades de elegir a quien vender los cueros era menor.

El estudio de la circulación de los bienes pecuarios que llegaban a Montevideo entre septiembre de 1784 y marzo de 1790 reveló que el 54,3\% de los cueros eran transportados hasta la ciudad por los propios productores, mientras que un poco más del $28,6 \%$ ya habían sido comercializados en la campaña (BIANGARDI, 2015a, p. 69-70). Aunque había una gran cantidad de productores y comerciantes involucrados en la circulación de estos bienes, se puede observar que existió cierta concentración ya que 30 productores (que representan el $4 \%$ de los 924 individuos identificados como introductores de bienes pecuarios) introdujeron el $38 \%$ de los cueros (BIANGARDI, 2015a, p. 102). Esta concentración es considerable, no obstante, como se verá más adelante, este fenómeno se daba aún en mayor medida en la exportación de los cueros.

\section{Mercado urbano y almacenamiento}

Cuando los cueros llegaban a la ciudad eran parte de numerosas transacciones y almacenados para su posterior embarque, aunque algunos fueron consumidos en el lugar en distintos

\footnotetext{
${ }_{7}^{7}$ Sobre los distintos tipos de embarcaciones que surcaban los ríos de la región, véase: Reitano (2006).
} 
usos. Se desconoce gran parte de ese mercado urbano, principalmente por los problemas que plantean las fuentes fiscales para dar cuenta del mismo.

La dificultad que presentaba ejercer un control sobre el comercio interno determinó que la alcabala se cobrase a tiendas y pulperías por sumas fijas. De esta manera, es imposible conocer las transacciones de esos establecimientos y, por ende, las operaciones de compra-venta de cueros. Tampoco aparecen en los libros manuales de alcabala muchas de estas operaciones entre personas. Esto plantea inconvenientes a la hora de tratar de reconstruir una serie de precios de los cueros vacunos para el Río de la Plata.

Salvo por algunos indicios no se conoce el precio que se pagaba por los cueros en la campaña ni tampoco en la ciudad ni en Europa. De esta manera, no es posible seguir la evolución de los niveles de precio a través de las distintas coyunturas de retracción y expansión de la demanda (representada principalmente por el comercio ultramarino). Un problema adicional lo constituye el hecho de que el sistema de comercialización de este bien era diferente en el mercado exportador en relación al mercado interno. Así, mientras en la campaña las transacciones se hacían por unidad, las compras de cueros para embarcar se hacían por peso. En consecuencia, más allá de algunas suposiciones generales, no es posible saber cuánto le quedaba al productor del valor de ese bien, ni cuál era el costo de la intermediación, ni la ganancia que obtenía el comerciante dedicado a la exportación.

Al respecto, Azcuy Ameghino hace una estimación por la cual supone que la pesada de 40 libras tenía un precio de dos pesos y que el cuero podría haber sido comprado en la campaña a cuatro reales y el traslado a Montevideo costar otros cuatro reales. En consecuencia, de existir un intermediario éste puede haber obtenido un 100\% de ganancia (AZCUY AMEGHINO, 2002, p. 56). Sin embargo, esta estimación no parece acertada por varias razones. En primer lugar, el precio del cuero en la campaña a 4 reales es muy bajo. En los libros auxiliares de alcabala el precio de aforo oscilaba entre los 6 reales el cuero de vaca y los 8 o 9 reales el de toro. En general, se considera que el precio de aforo era menor al de mercado. Además, suponiendo que el precio de aforo fuese coincidente con el de mercado, no es lógico que los pulperos que compraban cueros en Maldonado para venderlos en Montevideo hayan pagado alcabala en la Caja Real de Maldonado por el precio al que lo iban a vender y no al que lo habían comprado. En consecuencia, es probable que el nivel de precios del mercado interno haya sido superior a lo que se cree. En segundo lugar, el cálculo de 4 reales por cuero de costo de transporte parece excesivo. Como ya se mencionó, un carro podía transportar entre 80 y 100 cueros. Por ende, siguiendo esa estimación el dueño de un carro podía obtener 40 o 50 pesos por viaje. Es decir, que con dos o tres viajes podía ganar lo mismo que cobraba un capataz de estancia en todo un año. En realidad, el costo del transporte tiene que haber sido menor. Por último, los datos disponibles indican que 16 reales por pesada era un precio alto que solamente se conseguía en algunas ocasiones. Por consiguiente, el costo de la intermediación fue considerablemente menor. 
Por otro lado, también resulta muy difícil estimar el consumo interno de los cueros. En esa época el pellejo de los animales se usaba para tantos destinos diferentes que se torna imposible de mensurar. Por ejemplo, lo cueros se utilizaban para impermeabilizar las bodegas de las embarcaciones ${ }^{8}$, para cubrir carretas, tapar ventanas en los ranchos, fabricar calzado y correas y todo tipo de recipientes y accesorios. En consecuencia, además de una mercancía exportable, los cueros también constituían un insumo fundamental para muchos artesanos.

El almacenamiento de los cueros, por su parte, presentaba el mismo problema que ya se mencionó para el caso de su transporte: se trataba de una mercancía de gran volumen. Dada la cantidad de cueros que llegaban diariamente a Montevideo y su tamaño y, teniendo en consideración que se trataba de una ciudad pequeña, era lógico que no se pudiesen almacenar en las casas de los comerciantes. En consecuencia, los cueros se guardaban en terrenos baldíos que eran denominados huecos.

Otro inconveniente relacionado con el almacenamiento estaba vinculado con el ataque de las polillas. El apolillamiento podía reducir considerablemente el valor de un cuero que habitualmente quedaba relegado a algunos destinos del consumo interno. Este problema no encontró solución hasta bien entrado el siglo XIX cuando comenzó a utilizarse el arsénico para combatir las polillas (GIBERTI, 1986).

\section{El comercio ultramarino y la exportación de cueros}

\section{Embarque y comercialización ultramarina}

Durante la segunda mitad del siglo XVIII se produjo un incremento de la salida de cueros desde el complejo portuario rioplatense. La importancia regional de este proceso no atañó solamente a los grandes comerciantes que tuvieron gran incidencia en esta actividad. Sino que fue un eje articulador a nivel regional, tanto en cuanto a la participación de actores en el proceso comercial (carga de los navíos, carpinteros, herreros, entre otros), la demanda de bienes y servicios destinados al mantenimiento de la tripulación y pasajeros que demandaba esta actividad (JUMAR et al., 2006), sino también en la incidencia de pequeños y medianos cargadores.

Establecer la cantidad total de cueros que partieron desde el complejo portuario es difícil de medir ya que la información con la que contamos es fiscal, y los impuestos que se debían pagar por el comercio ultramarino de cueros implicaron un complejo proceso a partir de determinar cada unidad con un peso fijo de $35 \mathrm{~kg}$. Esto trajo consigo que sea necesario el pesarlos y establecer el "romaneo" y una cantidad teórica de cueros que se tomó como el número efectivo

${ }^{8}$ Por ejemplo, para las carenas realizadas por las fragatas correo entre los años 1767 y 1802 se compraron un total de 12.919 cueros por valor de 12.912 pesos. Ver: Sandrín (2016, p. 156). El bajo precio de estos (menos de un peso por cuero de promedio) es indicativo de que en este caso se trata de cueros de inferior calidad, lo que es una característica del mercado interno en general ya que los de una calidad superior se reservaban para la exportación.

${ }^{9}$ Pesaje. 
de los mismos. Para establecer la variación que representó este proceso para determinar el total de cueros que partieron se calculó en base a 7.226 cueros comprados por Juan de Llanos en el año 1805 . El peso de los cueros fue de $203.643 \mathrm{~kg}$ lo que nos da 5.818 unidades a 35, siendo necesario 1,2 cueros para llegar a la unidad de medida ${ }^{10}$. De esta manera, se da un incremento de la cantidad de cueros reales que partieron al comercio por sobre el total de unidades de $35 \mathrm{~kg}$, lo que permite ajustar la dinámica comercial ya que, por ejemplo, a la salida de cueros desde Montevideo para el período 1779- 1799 es necesario sumarle 882.926 cueros más.

Cuadro 1- Total de cueros por unidades y por romaneo salido por Montevideo 1779- 1799

\begin{tabular}{|c|c|c|}
\hline & Total de cueros & Por año media \\
\hline Por peso & 4.414 .630 & $220.731,5$ \\
\hline Por unidad & 5.297 .556 & $264.877,8$ \\
\hline Variación & 882.926 & $44.146,3$ \\
\hline
\end{tabular}

Fuentes: Elaboración propia a partir de AGN Sala XIII: 15- 10-3; 37-6-4; 37-10-5; 37-10-6-; $38-8-3$; 38-8-4; 38-1-1; 38-1-3; 38-2-3; 38-3-4; 38-4-4; 38-5-3; 38-6-4; 38-1-3; 39-1-3; 39-2-1; 39-22; 39-3-1; 39-3-2; 39-3-3; 39-4-3; 39-5-4; 39-6-2; 39-8-4; 39-9-3; 39-10-1; 39-10-4; 39-7-4; 15-10-3; $37-9-3$; 37-9-4; 38-10-3; 38-6-3. 14-9-5; 14-10-1; 14-10-2; 14-10-3; 14-10-4; 15-10-1; 15-10-2; 15-10-3.

Ahora bien, cómo fue la dinámica de salida de cueros desde el complejo portuario rioplatense y en particular desde Montevideo. La salida de cueros de los dos puertos habilitados de la Región Río de la Plata da un total de casi doce millones en estos veinte años y un promedio de casi seiscientos mil al año. Este importante número de cueros comercializados en el mercado ultramarino salió en forma relativamente equilibrada de dichos puertos, si bien desde Buenos Aires su número fue mayor que desde Montevideo durante el este período.

Cuadro 2- Totales de cueros salidos de Montevideo y Buenos Aires 1779- 1799 (en unidades)

\begin{tabular}{|c|c|c|c|}
\hline & Total de cueros & Porcentaje $\%$ & Por año media \\
\hline Montevideo & 5.297 .556 & 44,50 & $264.877,8$ \\
\hline Buenos Aires & 6.605 .022 & 55,49 & $330.251,1$ \\
\hline Total & 11.902 .578 & $100 \%$ & $595.128,9$ \\
\hline
\end{tabular}

Fuente: Véase cuadro 1

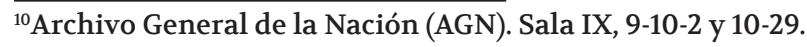


El movimiento naviero del complejo portuario rioplatense, durante el siglo XVIII, tuvo una dinámica progresivamente ascendente. La llegada y salida de los navíos legales comenzó a incrementarse en forma acelerada durante la década de 1760 y no volvió a bajar a las cifras de décadas previas durante el resto del período estudiado.

Durante la primera mitad del siglo XVIII, la división entre los destinos de las embarcaciones que arribaban a las costas rioplatenses se daba entre extranjeras y Sevilla- Cádiz. En general, eran embarcaciones de gran tamaño a la que solo podían acceder grandes comerciantes y consignatarios de casas comerciales afincadas en Cádiz. Con el correr de la segunda mitad del siglo esto fue variando notablemente, la circulación de navíos se hizo masiva, pequeñas embarcaciones lograron hacer mucho mas rentable cubrir el circuito con distintos puertos de la península española. Por este motivo, los pequeños y medianos comerciantes e industriales peninsulares se arriesgaron a enviar cantidades menores de manufacturas y la carga en el Río de la Plata fue ocupada por pequeños y medianos cargadores de cueros. En este contexto, la importancia del puerto de Cádiz en relación al complejo portuario rioplatense bajó notablemente como producto del empuje de otras regiones que vieron canalizadas sus necesidades en reglamentos que, desde la década de 1760, se redireccionaron para su beneficio. Los destinos de los cueros, se dividieron durante la segunda mitad del siglo XVIII, en tres partes: Cádiz, los puertos del Mediterráneo y los del Norte.

Se ha planteado que el cuero era un lastre conveniente para la circulación de los navíos ya que las piedras que se utilizaban habitualmente para ello tenían un alto costo (producto de la falta crónica de ese material en la zona) y, entonces, se retornaba, con cueros para mantener el nivel de flotación (AMARAL, 1998). Por el contrario, se puede afirmar que el cuero constituyó un bien importante en el comercio ultramarino durante este período. Esto se torna evidente al observar el epistolario comercial de varios comerciantes11, los cuales no solo están muy pendientes de los precios y dinámicas de esta mercancía, sino que también, en varias oportunidades las prefieren antes que a los metales preciosos. Allí se percibe que el aumento de la demanda fue muy importante, ya que era un insumo imprescindible para la industrialización.

La mayoría de los cueros, luego de su llegada al puerto de la península española, eran reenviados a otros lugares de Europa. Los arribados a Santander, por ejemplo, eran reenviados en su mayoría a la Bayona francesa, Burdeos, Ostende y Londres (MIGUEL LÓPEZ, 1996). Con lo cual, el circuito que comienza con la producción en la Región Río de la Plata cubre un intrincado recorrido hasta su final utilización en alguna maquina textil o algún otro destino de un centro industrial europeo.

\section{Cargadores}

La participación de la población de las ciudades del complejo portuario rioplatense en el desarrollo del comercio del cuero fue muy numerosa. En primer lugar, tenemos a los que

${ }^{11}$ Aria de la Cauta de Barcelona. Fondo Comercial. Ignacio Parera y Cía. Cartas, p. 16-17. 
participaron como peones, conductores de las pequeñas embarcaciones que transportaban los cueros a los navíos ultramarinos y estibadores, entro otros. En segundo lugar, se encuentran los cargadores/comerciantes que conectaban los mercados internos con los ultramarinos. Por último, había actores que indirectamente se beneficiaron del alto número de cueros comercializados como consecuencia del incremento de la actividad económica regional.

El proceso de carga de los cueros a las embarcaciones era una dinámica que era desarrollado por varias personas y que duraba semanas. Por ejemplo, el 15 de junio de 1804 se finalizó la carga de mil cueros en una embarcación ultramarina, hecha a cuenta de Juan de Llanos. Para ello se contrató a tres peones para pesar y apilar los cueros y seis peones para acarrear a bordo. Este procedimiento representó el pago de 15 pesos y 6 reales por ese trabajo que se le sumó al coste total.

Los cargadores de cueros eran aquellos actores que lograban conectar la producción de cueros con los intermediarios de la oferta de cueros regional y la demanda de los mercados ultramarinos. En la mayoría de los casos el comercio de cueros se producía por mercancías europeas que posteriormente intercambiaban por metales preciosos en el interior americano. Era una actividad con un margen de ganancia que permitió a aquellos que lo lograron hacer en grandes cantidades y en un periodo de tiempo medio o largo un éxito comercial.

En el Gráfico 1 se puede observar el incremento del número de cargadores que se dedicaron al comercio ultramarino de cueros entre 1720 y 1790. A partir de la década de 1750 comenzaron a llegar nuevos actores con redes de distintas regiones de la península española, los cuales se vincularon específicamente al comercio de cueros como consecuencia del incremento de la demanda externa de esta mercancía. Por ello, aumentó el número de actores dedicados a esta

Gráfico 1- Total de cargadores de cueros del complejo portuario rioplatense (1720 - 1799).

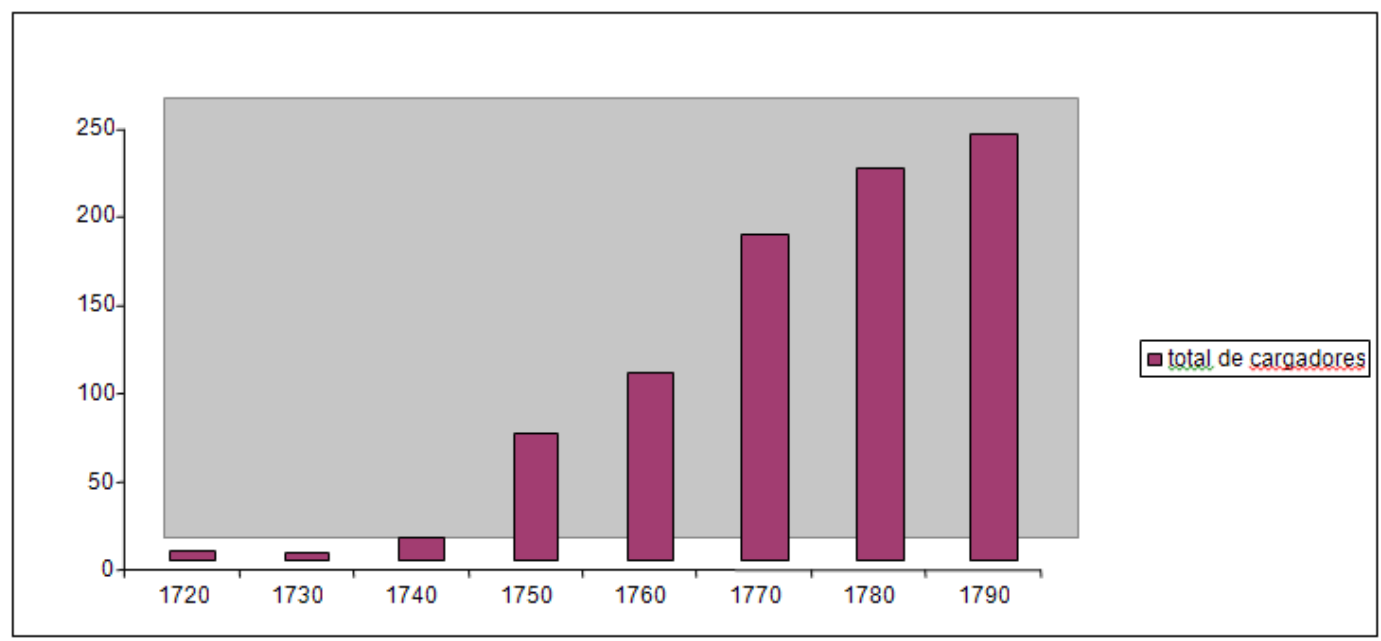

Fuente: Véase cuadro 1 
actividad durante las décadas siguientes que, además, se vio reforzada con las transformaciones institucionales que permitieron que no fuera necesario el paso por Cádiz como nexo obligatorio para el comercio de cueros.

La llegada de las fragatas correos desde mediados de la década del sesenta y el Reglamento y Aranceles Reales para el Comercio Libre de España a Indias fueron las respuestas de la Corona al proceso económico regional y global abierto, dando su apoyo a estos actores, restringiendo el peso de los monopolistas12 en el comercio de esta mercancía. Un cuestionamiento en este sentido, es el posible regularización de una actividad que ya venían desarrollando, esta posibilidad es concreta pero difícil de comprobar.

Al observar el movimiento de personas revelado por las fuentes se vislumbra que, en la mayoría de los casos, tras la llegada al Río de la Plata comienzan a comerciar como comisionistas de alguna casa y envían remesas de metales preciosos. Unos años después, empiezan progresivamente a incorporar envíos de cueros, que con el tiempo se incrementa en detrimento de los metales preciosos. El aumento de la competencia de quienes comercializaban manufacturas europeas a partir de la apertura progresiva de puertos y de la especialización de más regiones en el área, produjo un cambio en cuanto a la correlación de negociación entre aquellos que enviaban las mercancías desde la península española y quienes debían venderlas en la región Río de la Plata u otra región americana.

El número total de cargadores que intervinieron entre 1779 y 1799 fue de 1.386 , lo que nos habla de que una parte importante de la población se dedicó, en mayor o menor medida, a esta actividad. Esto permite ver la falta de monopolio en cuanto al acceso al comercio. Es decir, un gran número de pobladores tuvieron el contacto con productores y consumidores/ intermediarios ultramarinos gracias a la facilidad de cargar en forma legal cueros y a la llegada masiva de navíos destinados para ese fin. Como se puede observar en el cuadro 3, algo más de dos tercios de los cargadores operaban desde Montevideo13. Esto probablemente estuviera dado como producto de una permeabilidad mayor al pequeño comerciante por el reciente poblamiento de la ciudad y la cercanía entre el puerto y las zonas de producción de cueros. En el caso de Buenos Aires, dado que las zonas de producción se encontraron más alejadas y al tener una estructura comercial con mayor articulación, el número de actores intervinientes fue menor.

Cuadro 3- Totales de cargadores de Montevideo y Buenos Aires (1779-1799)

\begin{tabular}{|c|c|}
\hline Puerto & Cargadores \\
\hline Montevideo & 928 \\
\hline Buenos Aires & 458 \\
\hline Total & 1386 \\
\hline
\end{tabular}

Fuente: Véase cuadro 1

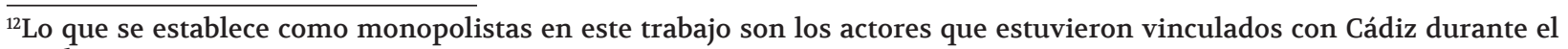
siglo XVIII.

${ }^{13}$ En este caso dividimos el análisis entre los dos puertos también, para complejizar el análisis y establecer las diferencias y similitudes entre los dos puertos más importantes y determinar las zonas de producción.
} 
La cantidad total de cueros que enviaron cada uno de los cargadores varió notablemente, como se observa en el cuadro 4. Por un lado, tenemos que el mayor número de cargadores, más de mil, enviaron menos de cinco mil cueros y, sumados, representaron algo más de un catorce por ciento del total de cueros exportados. Por otro lado, durante el período sólo treinta y cuatro cargadores enviaron más de cincuenta mil cueros y concentraron algo más de la mitad del comercio de cueros. Esto da cuenta de una gran concentración del comercio en muy pocas manos y, si bien el número de cargadores fue alto, la mayoría no tuvo una participación importante en el comercio.

Cuadro 4- Total de Cargadores de Buenos Aires y Montevideo según cantidad de cueros comercializados (1779-1799)

\begin{tabular}{|c|c|c|c|c|}
\hline & \multicolumn{2}{|c|}{ Cargadores } & \multicolumn{2}{c|}{ Cueros } \\
\hline - de 1.000 & 616 & $44,5 \%$ & 344.739 & $3,5 \%$ \\
\hline - de 5.000 & 499 & $36,1 \%$ & 1.107 .949 & $11,2 \%$ \\
\hline - de 10.000 & 110 & $7,8 \%$ & 813.856 & $8,2 \%$ \\
\hline - de 50.000 & 126 & $9,1 \%$ & 2.640 .023 & $26,6 \%$ \\
\hline + de 50.000 & 34 & $2,5 \%$ & 5.012 .248 & $50,5 \%$ \\
\hline Total & 1.386 & $100 \%$ & 9.918 .815 & $100 \%$ \\
\hline
\end{tabular}

Fuente: Véase cuadro 1

En este sentido, se puede observar que un grupo determinado se mantuvo como núcleo del comercio de cueros, estos cargadores bajaban la actividad mercantil por las circunstancias adversas, pero la mantenían en el tiempo. A su vez, se encuentra otro grupo mayoritario, fluctuante, que a partir de las oportunidades que se brindaban con la reapertura comercial, actuaban de forma intermitente. 
Cuadro 5- Total de cargadores de Montevideo según cantidad de cueros comercializados (1779-1799)

\begin{tabular}{|c|c|c|c|c|}
\hline & \multicolumn{2}{|c|}{ Cargadores } & \multicolumn{2}{c|}{ Cueros } \\
\hline - de 1.000 & 511 & $55,0 \%$ & 171.344 & $3,9 \%$ \\
\hline - de 5.000 & 294 & $31,7 \%$ & 642.310 & $14,5 \%$ \\
\hline - de 10.000 & 55 & $5,9 \%$ & 407.558 & $9,2 \%$ \\
\hline - de 50.000 & 54 & $5,9 \%$ & 1.072 .237 & $24,3 \%$ \\
\hline+ de 50.000 & 14 & $1,5 \%$ & 2.121 .181 & $48,1 \%$ \\
\hline Total & 928 & $100 \%$ & 4.414 .630 & $100 \%$ \\
\hline
\end{tabular}

Fuente: Véase cuadro 1

Para el caso de Montevideo, en donde se encontraba el número mayor de cargadores ${ }^{14}$, dos tercios del total, la concentración del comercio de los cueros fue altísima, sólo 14 comerciantes concentraron casi la mitad y 805 el 17\% de la cantidad de cueros. Como ya se mencionó, la cercanía con las zonas de producción de cueros probablemente permitió a una parte importante de la población con algún vínculo con la península española, el envío de pequeñas cantidades de cueros, sin el mantenimiento de un vínculo permanente como forma de pago de mercancías enviadas previamente o para el pago de deudas contraídas.

En síntesis, si se tienen en cuenta todas las actividades que involucraban la producción, circulación y exportación, que se han ido describiendo, es lógico suponer que en su conjunto estas tenían un peso importante en el nivel de ocupación de los habitantes del Río de la Plata. Para eso basta comparar los 924 productores y comerciantes y los 928 exportadores de Montevideo, mencionados en este trabajo, con los datos demográficos de esa ciudad brindados por Raquel Pollero. De acuerdo a esa información, en el año 1799 la ciudad de Montevideo y su campaña podían tener 12.034 habitantes en total (POLLERO, 2013), a lo que habría descontarle aproximadamente un $40 \%$ que serían los menores de 14 (POLLERO, 2013) y quedarían 7.221 pobladores económicamente activos. De los que, dada la alta tasa de masculinidad, solamente un $40 \%$ eran mujeres15. Es decir que se puede estimar a grosso modo que pudo haber 4.332 hombres adultos. En consecuencia, las 1.852 personas que aparecen en las bases de datos utilizadas para este trabajo produciendo

${ }^{14} \mathrm{El}$ alto número de cargadores que realizaron su operación en Montevideo no acredita que sean vecinos de la localidad, podrían ser del espacio regional, comerciantes asentados provisoriamente o capitanes de navíos. Para determinar que un número importante de estos cargadores se mantuvo un periodo relativamente prolongado en la región, se tomó algunos cargadores al azar y se rastreó su participación. El análisis puntilloso de todos los actores, es una tarea a futuro que realizaremos mediante el estudio de los padrones militares y demás fuentes que den cuenta de la totalidad, o gran parte, de la población que se encontraba en la región.

${ }^{15} \mathrm{El}$ índice de masculinidad para el año 1778 era de 140,2. 
o comercializando cueros representan el $42 \%$ de esos hombres adultos considerando el total de población del último año del período analizado, con lo que sin duda constituiría un porcentaje mayor para los años previos16. Esto sin contar los peones, transportistas y artesanos que constituían otro número importante de personas. Es lógico, entonces, suponer que la mayor parte de la población de Montevideo estaba, o había estado, vinculada de alguna manera al negocio del cuero. Tambien hay que rescatar que era una sociedad en donde una gran parte de los actores desarrollaron pluriactividades.

\section{Consideraciones finales}

En este artículo se analizó el complejo proceso de producción y comercialización de los cueros desde la extracción hasta su utilización como insumo industrial. En particular, el objetivo estuvo centrado en el desarrollo de las actividades económicas llevadas a cabo por los rioplatenses en la producción y comercialización de los cueros. Una primera observación en torno a la incidencia regional se observa en relación a la masiva participación, en mayor o menor medida, de gran parte de la población en el proceso del cuero. A partir del cruzamiento de los datos sobre la población y los expuestos sobre la producción y comercialización, es posible afirmar que el negocio del cuero en su conjunto constituía el eje de la economía regional, o por lo menos disputa esa primacía. En consecuencia, el incremento de la producción corambrera durante la segunda mitad del siglo XVIII tuvo un lugar destacado en el crecimiento económico de la región que se hizo evidente en el aumento demográfico (producto en gran parte de la migración atraída por las oportunidades comerciales), la ocupación de nuevas tierras y la expansión de todos los procesos productivos.

También se desprende de la cadena de producción y comercialización analizada en este trabajo la existencia de especializaciones laborales que podrían llevar a definir una serie de categorías ocupacionales entre los distintos agentes económicos que aparecen, aunque en el espacio disponible en este texto no fue posible detenerse en clasificaciones detalladas. De todas maneras, es importante destacar esto ya que a partir de una serie de estudios de casos sobre algunos comerciantes quedó la impresión de que esta economía se caracterizaba por la diversificación de inversiones, debido a que éstos evitaban la especialización como una estrategia para reducir el riesgo. Sin embargo, esta opción solamente era posible para aquellos que disponían del capital necesario, que sin duda constituían una minoría dentro de la población rioplatense. Sería necesario profundizar en estudios de caso de actores de los sectores subalternos que nos permita profundizar más en las potenciales diversas actividades en la que estos grupos participaban.

Finalmente, los datos sobre los actores, dan cuenta de una sociedad en donde el acceso al mercado, tanto en relación a los productores para llevar sus cueros a comercializar como los comerciantes para poner sus cueros en los navíos ultramarinos, era de una relativa facilidad dado que se desarrolló en pequeñas, medianas y grandes cantidades.

\footnotetext{
${ }^{16}$ En 1779 la población de Montevideo y su campaña era de apenas de 5.795 habitantes.
} 


\section{Referências}

AMARAL, Samuel. The rise of capitalism on the pampas: the estancias of Buenos Aires, 1785- 1870. Cambridge: Cambridge University Press, 1998.

AZCUY AMEGHINO, Eduardo. La otra historia: economía, estado y sociedad en el Río de la Plata colonial. Buenos Aires: Imago Mundi, 2002.

BIANGARDI, Nicolás. Una nueva área para la región: poblamiento y crecimiento económico en Maldonado (1755-1814). Revista Uruguaya de Historia Económica, Montevideo, v. 3, n. 4, p. 11-30, 2013.

BIANGARDI, Nicolás. Expansión territorial, producción ganadera y relaciones de poder en la región Río de la Plata: Montevideo y Maldonado a fines del siglo XVIII. 2015. Tesis (Doctorado en Historia) - UNLP, La Plata, 2015a.

BIANGARDI, Nicolás. Producción rural y fiscalidad en el Río de la Plata durante el siglo XVIII. Bibliographica Americana, Buenos Aires, n. 11, p. 52-68, $2015 \mathrm{~b}$.

CAMARDA, Maximiliano. La región Río de la Plata y el comercio ultramarino en las últimas décadas del siglo XVIII: circulación comercial, mercancías y actores. 2015. Tesis (Doctorado en Historia) - UNLP, La Plata, 2015.

DJENDEREDJIAN, Julio. Economía y sociedad en la Arcadia criolla: formación y desarrollo de una sociedad de frontera en Entre Ríos, 1750- 1820. 2003. Tesis (Doctorado en Historia) - UBA, Buenos Aires, 2003.

GARAVAGLIA, Juan Carlos. Pastores y labradores de Buenos Aires: una historia agraria de la campaña bonaerense 1700-1830. Buenos Aires: Ediciones de la Flor, 1999.

GELMAN, Jorge. Campesinos y estancieros: una región del Río de la Plata a fines de la época colonial. Buenos Aires: Los Libros del Riel, 1998.

GIBERTI, Horacio. Historia económica de la ganadería argentina. Buenos Aires: Hyspamérica, 1986. 
JUMAR, Fernando. La región Río de la Plata y su complejo portuario durante el Antiguo Régimen. In: FRADKIN, Raúl (Dir.). Historia de la provincia de Buenos Aires: Tomo 2. De la conquista a la crisis de 1820. Buenos Aires: Edhasa, 2012. p. 123-157.

JUMAR, Fernando. El primer boom de la exportación de cueros y la sociedad local. Río de la Plata. Fines del siglo XVII, comienzos del siglo XVIII. In: JORNADAS DE HISTORIA ECONÓMICA, 21., 2008, Caseros. Actas... Caseros, 2008.

JUMAR, Fernando. Le comerce atlantique au Río de la Plata 1680-1778. 2000. Tesis (Doctorado) EHESS, París, 2000.

JUMAR, Fernando; BIANGARDI, Nicolás. Espacio económico y territorialidad en el Río de la Plata del siglo XVIII. In: RICHARD-JORBA, Rodolfo; BONAUDO, Marta (Coord.). Historia regional: enfoques y articulaciones para complejizar una historia nacional. La Plata: Universidad Nacional de La Plata, 2014. p. 75-91.

JUMAR, Fernando et al. El comercio ultramarino y la economía local en el complejo portuario rioplatense. Siglo XVIII. Anuario IEHS, Tandil, n. 21, 2006.

MAYO, Carlos Alberto. Estancia y sociedad en la pampa (1740-1820). Buenos Aires: Biblos, 1995.

MIGUEL LÓPEZ, Isabel. El censo de manufacturas de 1784. Una nueva fuente para el análisis de la industria catalana. Revista Historia Económica, Madrid, v. 14, n. 1, p. 125-181, 1996.

MORAES, María Inés. Tendencias y coyunturas agrarias del litoral rioplatense, 1760-1800: un análisis cuantitativo. Investigaciones de Historia Económica, Madrid, v. 10, n. 1, p. 22-33, 2014.

MORAES, María Inés. Las economías agrarias del litoral rioplatense en la segunda mitad del siglo XVIII: paisajes y desempeños. 2011. Tesis (Doctorado) - UCM, Madrid, 2011.

MOUTOUKIAS, Zakarias. El crecimiento en una economía colonial de Antiguo Régimen: reformismo y sector externo en el Río de la Plata (1760-1796). Arquivos do Centro Cultural Colouste Gulbenkian, París, v. 34, p. 771-813, 1995.

PÉREZ, Osvaldo. Tipos de producción ganadera en el Río de la Plata Colonial. La estancia de alzados. En: AZCUY AMEGHINO, Eduardo et al. Poder terrateniente, relaciones de producción y orden colonial. Buenos Aires: Fernando García Cambeiro, 1996. 
POLLERO, Raquel. Historia demográfica de Montevideo y su campaña (1757-1860). 2013. Tesis (Doctorado) - UdelaR, Montevideo, 2013.

REITANO, Emir. Barcos y cargas. Los veleros del Plata durante el Período Colonial Tardío. In: JORNADAS DE HISTORIA ECONÓMICA,. 20, 2006, Mar del Plata. Actas Mar del Plata, 2006.

ROSAL, Miguel; SCHMIT, Roberto. Las exportaciones pecuarias bonaerenses y el espacio mercantil rioplatense (1768-1854). In: GARAVAGLIA, Juan Carlos; FRADKIN, Raúl (Ed.). En busca del tiempo perdido: la economía de Buenos Aires en el país de la abundancia 1750- 1865. Buenos Aires: Prometeo, 2004. p. 159-193.

SAGUIER, Eduardo. El mercado del cuero y su rol como fuente alternativa de empleo. El caso de trabajo a destajo en las vaquerías de la Banda Oriental. Revista de Historia Económica, Madrid, v. 9, n. 1, p. 103-126, 1991.

SARREAL, Julia. Globalization and the Guarani: from missions to modernization in the Eighteenth Century. 2009. Tesis (Doctorado), UH, Cambridge, 2009.

SILVA, Hernán Asdrúbal. El comercio entre España y el Río de la Plata. Banco de España, Servicio de Estudios. Estudios de Historia Económica, Madrid, n. 26, 1993.

SOVARZO, José; ERRECART, María Claudia. De tierra adentro al río. El complejo portuario rioplatense a partir de las guías de aduana de Buenos Aires a fines del siglo XVIII. In: JORNADAS INTERESCUELAS, 14., 2013, Mendoza. Actas Mendoza, 2013.

SANDRÍN, María Emilia. La demanda de bienes y servicios para la Corona y la navegación ultramarina en el complejo portuario rioplatense y la dinamización de la economía regional, 16801810. 2016. Tesis (Doctorado) - UNLP, La Plata, 2016. 